\title{
Quadrotor Aircraft Design based on the K60 Controller
}

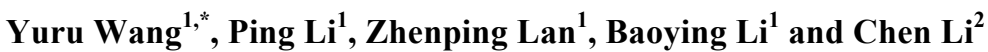

${ }^{1}$ School of Information Science and Engineering, Dalian Polytechnic University, Dalian 116034, China

${ }^{2}$ School of Electrical Engineering and Telecommunications, University of New South Wales, NSW 2052, Australia

Received 15 August 2017; Accepted 22 November 2017

\begin{abstract}
The stability of flight attitude is an important evaluation index of quadrotor aircraft applications. Attitude stability in particular plays an important role in micro-quadrotor aircraft control systems. Thus, a new quadrotor flight controller design based on the K60 processor is proposed to solve flight attitude inaccuracy, instability, and lag response problems. Quadrotor aircraft principles and the Newton-Euler equation were determined, and the mathematical model for miniaircraft motion was established. The Kalman filter principle was adopted to correctly analyze errors in measurement data. Subsequently, the proportional-integral-derivative (PID) cascade dual closed loop algorithm was established, and the stability of flight attitude control was analyzed. This study used experimental data to prove the correctness of the theoretical analysis. Results demonstrate that the quadrotor aircraft design effectively corrects system errors and inhibits lift fluctuation impact, thereby reducing attitude control fluctuation by $50 \%$. Without considering increase with time, the attitude estimation error is within $2^{\circ}$ and high-frequency control decreased to $-20 \mathrm{db}$. Therefore, this study realizes the rapid adjustment of flight attitude and improvements in flight stability. This study can provide significant references on system modeling, data processing, and flight control.
\end{abstract}

Keywords: Quadrotor aircraft, PID algorithm, Kalman filter, Flight attitude

\section{Introduction}

Quadrotor aircraft is a non-coaxial saucer-shaped aircraft. Given their simple structure, good mobility, and low cost, these aircraft types are widely used in civil and military applications, such as detection and reconnaissance [1]. The need for aircraft applications has increased in recent years, and related technical studies have been further developed. Several institutions have actively engaged in the study, design, and application of mini-aircrafts, in which study mainly focused on mechanical design, attitude flight control, and positioning navigation telecommunications, and so on. For instance, the Automation Laboratory of the Federal Institute of Technology in Lausanne (Switzerland) designed quadrotor aircrafts with indoor and outdoor autonomous flight [2].

Microelectronics and computer technologies have improved with the continuous development of integrated circuits. Subsequently, adapting to the working environment of aircraft has become increasingly rigorous, and the development of quadrotor aircraft in particular has gradually moved towards miniaturization and integration. Moreover, the requirements of flight accuracy, stability, and antiinterference have become complex. A micro-aircraft has lower development cost, simpler operation, higher safety, better flexibility, and better endurance than traditional aircraft. A micro-aircraft can also adapt to complicated environments. However, the requirements of a micro-aircraft are highly specific. Sample approximations include the

*E-mail address: haiian_wang@163.com

ISSN: 1791-2377 @ 2017 Eastern Macedonia and Thrace Institute of Technology. All rights reserved. doi:10.25103/jestr.106.04 following: component size $(6-20 \mathrm{~cm})$, quality build $(100 \mathrm{~g})$, valid load $(100 \mathrm{~g})$, task radius $(5 \mathrm{~km})$, flying height $(100 \mathrm{~m})$, and flight duration (20-60 minutes). In addition, the transformation time for attitude changed by a micro-aircraft is in the second level while attitude estimation error is approximately $2^{\circ}$. Furthermore, a micro-aircraft is affected by external interference; subsequently, complicated control technologies pose a major challenge to scholars [3].

Numerous studies have been conducted on the attitude estimation, control, and stability of micro-aircraft, as well as their relation to output torque linearization and interference factors [4-9]. However, the mini quadrotor aircraft is a complex nonlinear system with multiple inputs and outputs. Mini quadrotor aircraft systems require strong coupling between input and output variables. Constraints related to model and system non-integrality may cause uncertainties and external interference, which in turn may lead to higherlevel errors and control delays for the mini quadrotor aircraft system. Consequently, these constraints can affect the stability and precision of the flight system [10].

Based on the above considerations, quadrotor aircraft principles are analyzed in this study. A method is proposed for the mini quadrotor aircraft design based on the K60 controller. The aims of the design method are to obtain accurate attitude data and correct errors introduced by the sensors by employing an attitude algorithm, thereby improving the attitude stability of the mini quadrotor aircraft.

\section{State of the art}

The quadrotor aircraft, an electric autonomous aircraft, can take off vertically or land using multi-rotors. Due to its 
outstanding performance and unique flight technology, the quadrotor aircraft has been widely studied by scholars [11]. However, the control system of mini quadrotor aircraft is affected by many factors, such as nonlinearity and underactuation issues and undefined parameters; thus, satisfactorily controlling the flight attitude of quadrotor aircraft is difficult to achieve [12]. To solve these aircraft control problems, Chinese and international scientists have carried out relevant studies and considered several methods. Yan [13] and Rao [14] characterized the flight attitude while Liu [1] and $\mathrm{Wu}$ [10] studied the self-interference of quadrotor aircraft. Their study also described the impact of external uncertainties on control systems. Garcia [15] analyzed flight data using the Kalman filter method to establish the effectiveness of the Kalman filter algorithm. Xu [16] studied system response speed and conducted continuous processing of discontinuous variables to eliminate synovial control jitter, but system stability and freedom were not analyzed. Liu [17], Mahony, and Kumar [18] analyzed the satellite navigation system of quadrotor aircraft to determine flight attitude, speed, and position accuracy. However, their studies were not suitable for indoor flight or for areas with weak satellite navigation system signals. Das et al. [19] used the back-stepping method to design a controller. Although nonlinear system tracking for model accuracy was well realized, control performance was not considered. Qiang et al. [20] applied an optical flow sensor to realize the hovering stability of quadrotor aircraft in certain areas, but the errors caused by dark light or textured ground were not analyzed. He et al. [21] studied the flight attitude control of a quadrotor aircraft using visual algorithm. Lin [22] and Sun et al. [23] applied the synovial control and adaptive methods for quadrotor aircraft control. Although noise interference was effectively restrained, instantaneous jitters also occurred.

The above studies generally depend on the establishment of a system model; however, system models are often inadequate for practical applications. For instance, for an aircraft flight to meet duration standards, the motor and rotor transmission efficiency, aircraft power output efficiency, aircraft weight, and aircraft power and function modules require an integrated design. These integrated components can affect system modeling, and consequently, influence system stability and duration.

A cascade dual closed-loop proportional-integralderivative (PID) controller, which is not dependent on a system model, is designed by this study to solve the above problems. The PID controller can conduct real-time tracking for the fast adjustment of flight attitude. The system integrates Kalman filter into the inertial measurement data solution and transforms the quaternion Euler function to calculate the intermediate variable of the decoupling operation. The proposed method effectively decreases noise interference and reduces temperature drifts and the zero drift phenomenon. The startup Electronic Speed Control (ESC) program is designed for the nonlinear processing and quick response of motor outputs.

The remainder of this study is organized as follows: Section 3 describes the quadrotor aircraft principle; establishes the control model of the quadrotor aircraft; determines the solution of flight attitude and PID control algorithm; and describes the hardware and software design of the quadrotor aircraft. Section 4 presents the experimental measurement and result analysis. The last section summarizes the conclusions.

\section{Methodology}

\subsection{Overview of flight attitude}

The model of the quadrotor aircraft is mainly composed of a motor with a propeller, drive module, and motion controller (Fig. 1). The action of an aircraft generally depends on the rotation difference of four motors. The basic motion modes of the quadrotor aircraft are activated by roll, pitch, and yaw controls. These three modes are used as basis for changing the flight attitude and adjusting the position of the quadrotor aircraft. The differential rotational speed adjustment of Rotors 1 and 3 produces roll motion, whereas the differential rotational speed adjustment of Rotors 2 and 4 produces pitch motion. Yaw motion can be produced by improving (or decreasing) the speeds of Rotors 2 and 4 and decreasing (or improving) the speeds of Rotors 1 and 3 . In threedimensional space, the flight attitude of a quadrotor aircraft can be described by three Euler angles: roll angle $\varphi$, pitch angle $\theta$, and yaw angle $\psi$. As shown in Fig. 1, the three Euler angles are depicted by the intersection angles between earth-fixed axis system $\left(\mathrm{x}_{\mathrm{W}}, \mathrm{y}_{\mathrm{W}}\right.$, and $\left.\mathrm{z}_{\mathrm{W}}\right)$ and body axis system $\left(x_{B}, y_{B}\right.$, and $\left.z_{B}\right)$. When the quadrotor aircraft meets the origin of the body axis system and the geometric center and centroidal center of the quadrotor aircraft in the same position, the effects of air-flow velocity and air resistance are ignored during flight, and the tension of the quadrotor aircraft in each direction is proportional to the square of the propeller speed. The established model of translational and rotational motion of the quadrotor aircraft is built on the Newton-Euler equation [24-25].

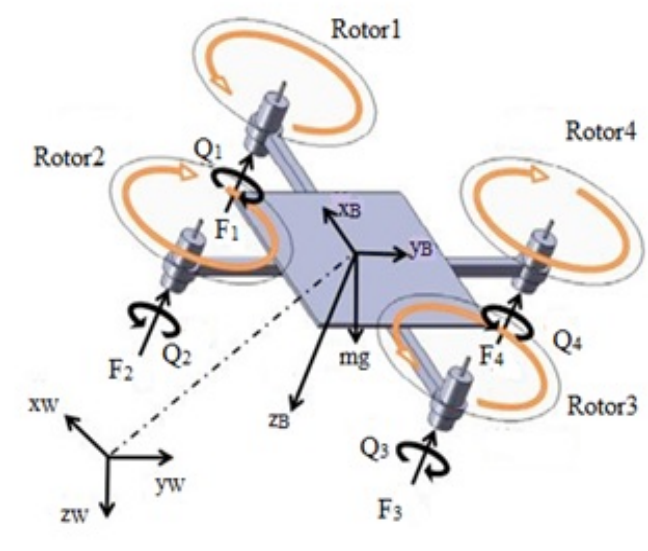

Fig. 1. Model of quadrotor aircraft

Through the two coordinate systems of the unit vector operation, the direction cosine vector matrix of the roll angle $\varphi$, pitch angle $\theta$, and yaw angle $\psi$ in earth-fixed axis system can be obtained as: 
$\mathrm{R}_{\mathrm{z}}(\varphi)=\left|\begin{array}{ccc}\cos \varphi & \sin \varphi & 0 \\ -\sin \varphi \cos \varphi & 0 \\ 0 & 0 & 1\end{array}\right| \mathrm{R}_{\mathrm{y}}(\theta)=\left|\begin{array}{ccc}\cos \theta & -\sin \theta \\ 0 & 1 & 0 \\ \sin \theta & 0 & \sin \theta\end{array}\right| \mathrm{R}_{\mathrm{x}}(\psi)=\left|\begin{array}{ccc}1 & 0 & 0 \\ 0 & \cos \psi & \sin \psi \\ 0-\sin \psi \cos \psi\end{array}\right|$

The rotation transformation matrix can be obtained as:

$$
\mathrm{C}(\theta, \varphi, \psi)=\left|\begin{array}{ccc}
\cos \theta \cos \varphi & \sin \psi \sin \theta & -\sin \theta \\
\cos \psi \sin \theta \sin \varphi-\sin \psi \cos \varphi & \sin \psi \sin \theta \sin \varphi+\cos \psi \cos \varphi & \cos \theta \sin \psi \\
\cos \psi \sin \theta \cos \varphi+\sin \psi \sin \varphi & \sin \psi \sin \theta \cos \varphi-\cos \psi \sin \gamma \varphi & \cos \theta \cos \psi
\end{array}\right|
$$

The total lifting force of the quadrotor aircraft is provided by four brushless motors. The total pulling force acting on the airframe is the sum of the four propeller forces given that coupling between propellers is non-existent. In this study, the revolution of the propeller is designated as $\omega$ and lift coefficient is represented by $\mathrm{C}$. Total lifting force $\mathrm{F}$ is expressed as:

$$
\mathrm{F}=\mathrm{F}_{1}+\mathrm{F}_{2}+\mathrm{F}_{3}+\mathrm{F}_{4}=\mathrm{C}\left(\omega_{1}^{2}+\omega_{2}^{2}+\omega_{3}^{2}+\omega_{4}^{2}\right)
$$

The torques $\mathrm{L}_{\mathrm{x}}$ (roll lift), $\mathrm{L}_{\mathrm{y}}$ (pitch lift), and $\mathrm{L}_{\mathrm{Z}}$ (vertical lift) generated by each axis are as follows:

$$
\begin{aligned}
& \mathrm{L}_{\mathrm{x}}=\operatorname{dc}\left(\frac{\sqrt{2}}{2} \omega_{1}^{2}-\frac{\sqrt{2}}{2} \omega_{2}^{2}-\frac{\sqrt{2}}{2} \omega_{3}^{2}+\frac{\sqrt{2}}{2} \omega_{4}^{2}\right) \\
& \mathrm{L}_{\mathrm{y}}=\operatorname{dc}\left(\frac{\sqrt{2}}{2} \omega_{1}^{2}+\frac{\sqrt{2}}{2} \omega_{2}^{2}-\frac{\sqrt{2}}{2} \omega_{3}^{2}-\frac{\sqrt{2}}{2} \omega_{4}^{2}\right) \\
& \mathrm{L}_{\mathrm{z}}=\mathrm{c}\left(\omega_{1}^{2}-\omega_{2}^{2}+\omega_{3}^{2}-\omega_{4}^{2}\right)
\end{aligned}
$$

Based on the above analysis, the PID controller is highly suitable for the flight attitude control model of the quadrotor aircraft.

\subsection{Mechanical-structural design of quadrotor aircraft}

Weight, arm length, and center of gravity are the main parameters considered in the mechanical-structural design of the quadrotor aircraft. In designing the frame, the lightweight index means high-flight efficiency. Thus, carbon fiber rod is considered as the main frame material for the quadrotor aircraft design. In terms of structure, the bridge arm of the frame should not be excessively short, as an excessively short bridge arm can affect propeller airflow and delimit aircraft control. Similarly, a long arm frame can increase the antitorque moment of the motor force and reduce quadrotor aircraft stability. In principle, an aircraft frame is 1.9-2.3 times the length of its propeller. Moreover, to significantly improve aircraft controllability, the frame structure should be symmetrical in order for the center of gravity to be near the center of the quadrotor aircraft. Thus, an F450 carbon fiber frame (i.e., hollow engineering plastic PA66 with $30 \%$ ultra-high-strength) is selected as the material for the study. Consequently, the quality of the quadrotor aircraft is relatively light and its strength is relatively high. The frame length of the quadrotor aircraft is only $12 \mathrm{~cm}$.

Quadrotor aircraft motors and propellers require a pairing selection. In this study, the hollow DC motor is considered for the quadrotor aircraft. The DC motor has outstanding energy saving, control, and drag characteristics.

Furthermore, modules 716,720 , and 820 are commonly adopted for mini quadrotor aircraft motors. In these modules, the first digit represents the motor diameter whereas the last two digits represent the length of the motor. For mini-sized motors, a large propeller decreases motor efficiency and causes serious motor heating. By contrast, a small propeller for large-sized motors leads to low-flight efficiency. Therefore, module 720 with $55 \mathrm{~mm}$ propeller is selected in this study, and the physical object is shown in Fig. 2.

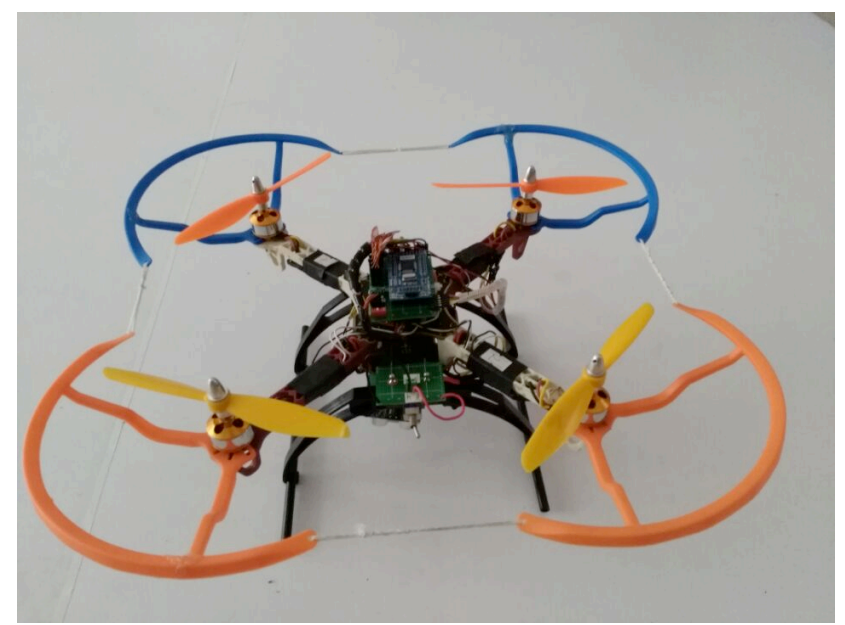

Fig. 2. Mini quadrotor aircraft physical object

\subsection{Analysis of dual closed-loop PID cascade control algorithm}

This study introduces angular velocity feedback to the control algorithm and adopts the working principle of single closed loop PID controls to establish the dual closed-loop PID cascade controller. However, interference is adjusted and subsequently eliminated in the inner loop by the dual closed-loop PID algorithm. In general, the anti-interference capability and robustness of dual closed-loop PIDs are stronger than those of single closed-loop PIDs.

The proposed system consists of three independent dual closed-loop PID controllers corresponding to the three attitude angles of the quadrotor aircraft. The similarity of the controlling structures on each channel is considered. Fig. 3 shows the dual closed-loop PID controller with pitch angle as an example. The input of the outer loop controller is pitch angle $\mathrm{w}_{\theta}$, the output is the expected value of angular velocity $U_{1 \theta}$, and the expected value (in degrees) of pitch angle is $r_{\theta}$. The expected value of the pitch angle can be obtained by positioning the quadrotor aircraft horizontally to take its 1,000 times measurement and by collecting angle data to calculate the median angle. The inner loop of the controller is an important system component because it affects flight attitude adjustment. The input of the inner loop is angular acceleration $\omega_{\theta}$; the output is in throttle increments of $\Delta \mathrm{U}_{2 \theta}$ (i.e., pulse-width-modulation (PWM) 
values in high voltages); and the expected value is the output of the outer loop $\mathrm{U}_{10}$.

The PID control algorithm adopts the incremental digital PID. Unlike in positional digital PID, the microprocessor only needs output control increments in incremental PID control, i.e., positional variations of the actuators. Therefore, the quadrotor aircraft can avoid serious accidents when control volume increases or decreases sharply and suddenly.
The output equation of the outer loop controller is given by Equation (5), where $\mathrm{K}_{\theta \mathrm{P}_{1}}, \mathrm{~K}_{\theta \mathrm{I}_{1}}$, and $\mathrm{K}_{\theta \mathrm{D}_{1}}$ are the proportional, integral, and differential coefficients of the outer loop. The output equation of the inner loop controller is given by Equation (6).

$$
\left\{\begin{array}{c}
\Delta \mathrm{U}_{1 \theta \mathrm{k}}=\mathrm{U}_{1 \theta \mathrm{k}}-\mathrm{U}_{1 \theta \mathrm{k}-1}=\mathrm{K}_{\theta \mathrm{P}_{1}} \Delta \mathrm{e}_{1 \theta \mathrm{k}}+\mathrm{K}_{\theta I 1} \mathrm{e}_{1 \theta \mathrm{k}}+\mathrm{K}_{\theta \mathrm{D} 1}\left(\Delta \mathrm{e}_{1 \theta \mathrm{k}}-\Delta \mathrm{e}_{1 \theta(\mathrm{k}-1)}\right) \\
\mathrm{e}_{1 \theta \mathrm{k}}=\mathrm{r}_{\theta \mathrm{k}}-\mathrm{w}_{\theta \mathrm{k}} \\
\mathrm{U}_{1 \theta \mathrm{k}}=\mathrm{U}_{1 \theta \mathrm{k}}+\Delta \mathrm{U}_{1 \theta \mathrm{k}}=\mathrm{K}_{\theta \mathrm{P}_{1}} \mathrm{e}_{1 \theta \mathrm{k}}+\mathrm{K}_{\theta \mathrm{I}_{1}} \sum_{\mathrm{i}=1}^{\mathrm{k}} \mathrm{e}_{1 \theta \mathrm{i}}+\mathrm{K}_{\theta \mathrm{D} 1}\left(\mathrm{e}_{1 \theta \mathrm{k}}-\mathrm{e}_{1 \theta(\mathrm{k}-1)}\right)+\Delta \mathrm{U}_{1 \theta \mathrm{k}}
\end{array}\right.
$$

$$
\left\{\begin{array}{c}
\mathrm{e}_{2 \theta \mathrm{k}}=\mathrm{U}_{1 \theta \mathrm{k}}-\omega_{\theta \mathrm{k}} \\
\Delta \mathrm{U}_{2 \theta \mathrm{k}}=\mathrm{U}_{2 \theta \mathrm{k}}-\mathrm{U}_{2 \theta(\mathrm{k}-1)}=\mathrm{K}_{\theta \mathrm{P}_{1}} \Delta \mathrm{e}_{2 \theta \mathrm{k}}+\mathrm{K}_{\theta \mathrm{\theta I}} \mathrm{e}_{2 \theta \mathrm{k}}+\mathrm{K}_{\theta \mathrm{D} 1}\left(\Delta \mathrm{e}_{2 \theta \mathrm{k}}-\Delta \mathrm{e}_{2 \theta(\mathrm{k}-1)}\right) \\
\mathrm{U}_{2 \theta \mathrm{k}}=\mathrm{U}_{2 \theta \mathrm{k}}+\Delta \mathrm{U}_{2 \theta \mathrm{k}}=\mathrm{K}_{\theta \mathrm{P}_{2}} \mathrm{e}_{2 \theta \mathrm{k}}+\mathrm{K}_{\theta \mathrm{I}_{2}} \sum_{\mathrm{i}=1}^{\mathrm{k}} \mathrm{e}_{2 \theta \mathrm{i}}+\mathrm{K}_{\theta \mathrm{D} 2}\left(\mathrm{e}_{2 \theta \mathrm{k}}-\mathrm{e}_{2 \theta(\mathrm{k}-1)}\right)+\Delta \mathrm{U}_{2 \theta \mathrm{k}}
\end{array}\right.
$$

Where $\mathrm{K}_{\theta \mathrm{P}_{2}}, \mathrm{~K}_{\theta \mathrm{I}_{2}}$, and $\mathrm{K}_{\theta \mathrm{D}_{2}}$ are the proportional, integral, and differential coefficients of the inner loop.

The output value of the inner loop is limited to the throttle (Fig. 3) to avoid an overshoot (i.e., excessive output) phenomenon. System throttle is usually controlled from -100 to +100 microseconds. The controls of the yaw and roll angles are similar to the control of the pitch angle. The output of attitude data at each moment is calculated by Equations (5) and (6).

The three Euler angles of the system are controlled by flight attitude calculation and PID controller calculation. The output data of the throttle is obtained after limiting the output. This study has established the dynamics model of the quadrotor aircraft in Section 3.1. The relationship of the four rotors outputs can be expressed by Equation (7).

$$
\left\{\begin{array}{l}
\mathrm{U}_{1}=\mathrm{U}_{2 \varphi}-\mathrm{U}_{2 \theta}-\mathrm{U}_{2 \psi} \\
\mathrm{U}_{2}=\mathrm{U}_{2 \varphi}-\mathrm{U}_{2 \theta}+\mathrm{U}_{2 \psi} \\
\mathrm{U}_{3}=\mathrm{U}_{2 \varphi}+\mathrm{U}_{2 \theta}+\mathrm{U}_{2 \psi} \\
\mathrm{U}_{4}=\mathrm{U}_{2 \varphi}+\mathrm{U}_{2 \theta}-\mathrm{U}_{2 \psi}
\end{array}\right.
$$

As depicted in Equation (7), each flight attitude can determine the resultant interaction of the four rotors. The lifting force of each rotor is the resultant force of three Euler angles and PID control output.

\subsection{Application principle of Kalman filter}

Accurate attitude measurement data is important for flight stability. The size and lightweight characteristics of a mini quadrotor aircraft can easily cause large deviations in measurement data (e.g., disturbance torque) resulting from external environment interference. Thus, a filter is added to the feedback link to control the influence of disturbance torque on the controller. Given its capability to perform higher-level calculation in real time, the Kalman filter is considered and combined with the hardware design of the mini quadrotor aircraft. The Kalman filter requires that the quadrotor aircraft system is a stochastic linear discrete system. The linear stochastic differential equation is:

$$
\left\{\begin{array}{c}
\mathrm{X}_{\mathrm{k}}=\varphi_{\mathrm{k} \mid \mathrm{k}-1} \mathrm{X}_{\mathrm{k}-1}+\theta_{\mathrm{k} \mid \mathrm{k}-1} \mathrm{~W}_{\mathrm{k}-1} \\
\mathrm{Z}_{\mathrm{k}}=\mathrm{H}_{\mathrm{k}} \mathrm{X}_{\mathrm{k}}+\mathrm{V}_{\mathrm{k}}
\end{array}\right.
$$

Where $\mathrm{H}_{\mathrm{k}}$ represents a parameter of the measurement system; $\mathrm{Z}_{\mathrm{K}}$ denotes measured value; $\varphi_{\mathrm{k}}, \theta_{\mathrm{k}}$, and $\mathrm{w}_{\mathrm{k}-1}$ are system parameters; and $\mathrm{V}_{\mathrm{k}}$ are system-correlated white noise sequences. However, $\mathrm{V}_{\mathrm{k}}$ belongs to white noise sequence in the angular velocity feedback system. Thus, the system utilizes Kalman filter when the white noise sequences are transformed. The white noise of colored noise is usually realized by information amplification measurements. Colored noise $\mathrm{V}_{\mathrm{k}}$ can be approximated as:

$\mathrm{V}_{\mathrm{k}}=\psi_{\mathrm{k} . \mathrm{k}-1} \mathrm{~V}_{\mathrm{k}-1}+\xi_{\mathrm{k}-1}$

The whitening vector expression of process noise in the angular velocity feedback system can be obtained by Equations (8) and (9) [26]:

$$
\dot{\mathrm{Z}}_{\mathrm{k}}=\dot{\mathrm{H}}_{\mathrm{k}} \mathrm{X}_{\mathrm{k}}+\dot{\mathrm{V}}_{\mathrm{k}}
$$

In the quadrotor aircraft design, the current generated by the motor can be deduced by the speed of motor feedback, and the predictive value and estimated covariance of angular velocity $\omega=\left[\begin{array}{lll}\mathrm{q} & \mathrm{p} & \Gamma\end{array}\right]^{\mathrm{T}}$ in Kalman filter can be obtained as:

$\left\{\begin{array}{c}\hat{\omega}_{\mathrm{k} \mid \mathrm{k}-1}=\hat{\omega}_{\mathrm{k}-1}+\mathrm{B}_{\mathrm{k}} \mathrm{U}_{\mathrm{k}}\left(\mathrm{f}_{1, \mathrm{~L}} ., \mathrm{f}_{4}\right) \\ \mathrm{P}_{\mathrm{K} \mid \mathrm{K}-1}=\mathrm{P}_{\mathrm{K}-1 \mid \mathrm{K}-1}+\mathrm{Q}_{\mathrm{K}}\end{array}\right.$

The optimal Kalman gain can be obtained by the state transition matrix $\mathrm{F}_{\mathrm{k}}=\mathrm{I}$ : 


$$
\mathrm{Kg}=\frac{\mathrm{P}_{\mathrm{k} k \mathrm{k}-1}\left(\dot{\mathrm{H}}_{\mathrm{k}}\left(\mathrm{f}_{1}, \cdots, \mathrm{f}_{4}\right)\right)^{\mathrm{T}}}{\left.\dot{\mathrm{H}}_{\mathrm{k}}\left(\mathrm{f}_{1}, \cdots, \mathrm{f}_{4}\right)\right) \mathrm{P}_{\mathrm{k} k \mathrm{k}-1}\left(\dot{\mathrm{H}}_{\mathrm{k}}\left(\mathrm{f}_{1}, \cdots, \mathrm{f}_{4}\right)\right)^{\mathrm{T}}+\dot{\mathrm{R}}_{\mathrm{k}}\left(\mathrm{f}_{1} \cdots, \mathrm{f}_{4}\right)}
$$

The state estimation and covariance estimation are:

$$
\begin{aligned}
& \widehat{\omega}_{k}=\widehat{\omega}_{k \mid k-1}+K_{k}\left(Z_{k}-\dot{\mathrm{H}}_{\kappa}\left(f 1, \ldots, f_{4}\right) \widehat{\omega}_{k \mid k-1}\right) \\
& P_{K \mid K-1}=\left(I-K_{k} \dot{H}_{k}\left(f 1, \cdots, f_{4}\right)\right) P_{\mathrm{k} \mid k-1}
\end{aligned}
$$

The Kalman filter recursive equation is composed of Equations (11), (12), and (13). Based on the analysis of Section 3.1, Equation (14) can be obtained, and the relationships between the angular velocity of filtering, the interfere of disturbance torque $\hat{\omega}_{\mathrm{k}}=[\hat{\mathrm{P}}, \hat{\mathrm{Q}}, \hat{\mathrm{R}}]^{\mathrm{T}}$, and the change rate of Euler angle $\dot{\eta}=[\dot{\varphi}, \dot{\theta}, \dot{\psi}]^{\mathrm{T}}$ :

$$
\left|\begin{array}{c}
\varphi \\
\dot{\theta} \\
\dot{\psi}
\end{array}\right|=\left|\begin{array}{ccc}
1 & \sin \varphi \tan \theta & \cos \varphi \tan \theta \\
0 & \cos \varphi & -\sin \varphi \\
0 & \frac{\sin \varphi}{\cos \theta} & \frac{\cos \varphi}{\cos \theta}
\end{array}\right|\left|\begin{array}{c}
\hat{P} \\
\hat{Q} \\
\hat{R}
\end{array}\right|
$$

Where $\dot{\eta}$ is substituted to operate in a dual closed-loop PID controller, and the output torque of motors can then be obtained.

\subsection{Hardware design of quadrotor aircraft}

Fig. 4 shows that the hardware of the system is mainly composed of a microcontroller unit (MCU), sensors, ESC, WiFi telecommunication, and power supply. MCU is the core module of the quadrotor aircraft control system. MCU collects information from sensors, controls the attitude angle in real time, corrects errors, controls the speed of four motors, and transmits data. Therefore, K60DN512VLL10 designed by Freescale Semiconductor is selected to be the core controller chip. The system clock frequency of the chip can reach $200 \mathrm{MHz}$. The K60DN512VLL10 controller chip can effectively ensure the system that operates in the program running speed, and K60DN512VLL10 can obtain relevant adjustment parameters to adjust the flight attitude in real-time. Thus, the quadrotor aircraft can operate stably and reliably. The chip has the advantages of low power consumption, high performance, and high precision. K60DN512VLL10 has multiple functions of the fast multichannel 16-bit AD conversion, DA conversion, and a programmable-gain operational amplifier. The rich PWM resources are enough to control four-way brushless motors.

The accuracy of the quadrotor aircraft depends largely on the accuracy of the sensors. Therefore, MPU6050 (high precision gyroscope accelerometer) is selected to collect the angular velocity and the acceleration of the quadrotor aircraft. The static accuracy of the MPU6050 angle measurement reaches $0.05^{\circ}$, and the dynamic accuracy reaches $0.1^{\circ}$. The data output frequency is up to $100 \mathrm{~Hz}$, and the baud rate is $115200 \mathrm{bps}$. All status data can be renovated every 10 millisecond. Moreover, MPU6050 automatically corrects the accelerometer by software operation and corrects the angular measurement errors caused by acceleration zero drift. This module can correct the zero drift of the gyroscope automatically when MPU6050 works in the stationary state.

The system selects 30 A brushless ESC designed by Xinxida Co., Ltd. Under the limit condition, the sustainable current is $30 \mathrm{~A}$ and can reach $35 \mathrm{~A}$ at an instant, $40 \mathrm{~A}$ can last for 10 seconds. The signal frequency range of the throttle is between 50 and $432 \mathrm{~Hz}$. The speed of motors is up to $2100 \mathrm{rpm}$. The voltage range is between 4 and $16 \mathrm{~V}$. It has under voltage protection, over-voltage protection, and over temperature protection.

The wireless module is the interface between the host computer and the quadrotor aircraft, which is used to transmit relevant parameters to view and modify the flight state. This study selects a $2.4 \mathrm{G}$ wireless serial port USRWIFI232-T module with $100 \mathrm{~mW}$. The transmission distance is up to 280 meters, and USB-WIFI232-T supports the frequency-hopping mode and switching channel automatically to effectively avoid the burst interference. The built-in wireless telecommunication algorithm has the advantages of precise time division and synchronization to ensure that the module has the abilities of data transmission, packet loss retransmission, and error correction, which can improve telecommunication reliability. The telecommunication process of the system is shown in Fig. 5. The MCU data are collected and transmitted to the WiFi module. They are displayed on the handheld devices. The current testing data are shown on the screen.

\subsection{Software design of quadrotor aircraft}

The rationality of the software design affects the response speed of the system, especially for the mini quadrotor aircraft. The output response speed is also a factor that affects the stability of the quadrotor aircraft. The diagram of software design of this study is shown in Fig. 6. The initialization of the system includes the initialization of the $\mathrm{I} / \mathrm{O}$ port, the initialization of the system clock and system parameters. Peripheral initialization includes the initialization of MPU6050, electrical control, and serial port. The detection procedure and the communication status of the quadrotor aircraft, which flies in the air, are completed through the self-test program. Quadrotor aircraft attitude control includes pitch, yaw, and roll control. The system collects the attitude parameters, which are integrated with data to obtain the flight parameters. The Kalman filter is used to correct the flight parameters' errors. The attitude algorithm and the PID operation are carried out to obtain the output torque of the rotor. Thus, the system can control the flight attitude, and the response time of the program is at the nanosecond level.

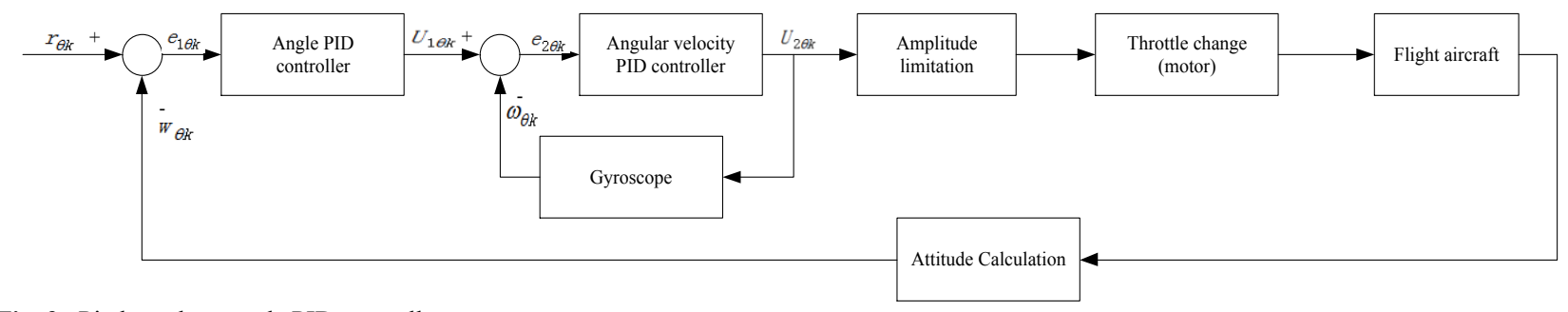

Fig. 3. Pitch angle cascade PID controller 


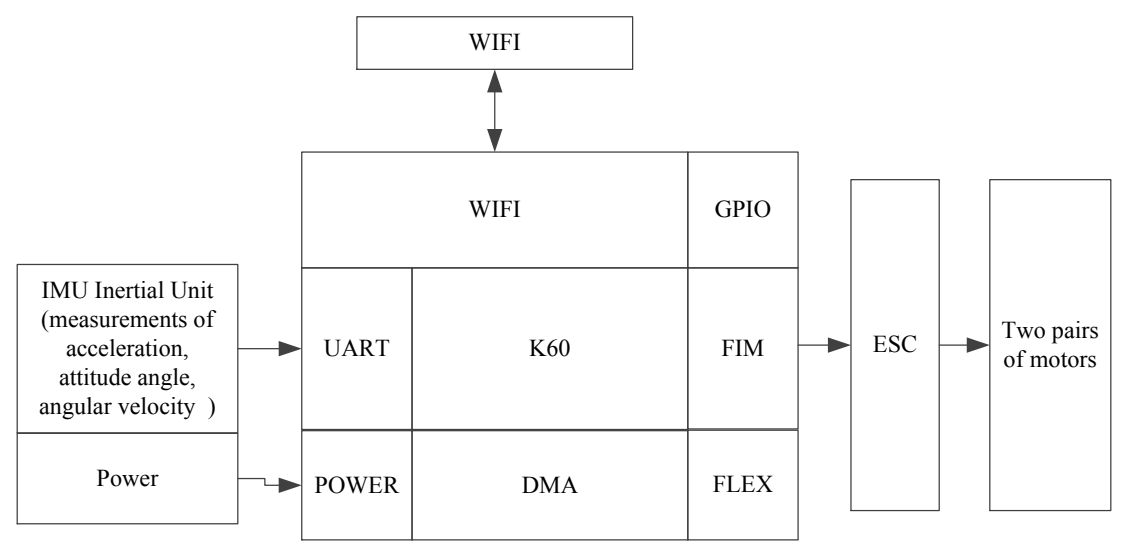

Fig. 4. Hardware Design Structural Diagram

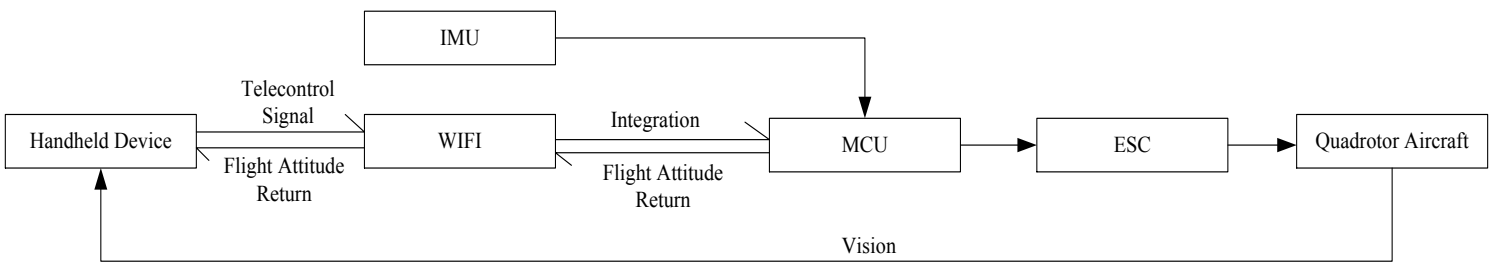

Fig. 5. Diagram of wifi communication signal

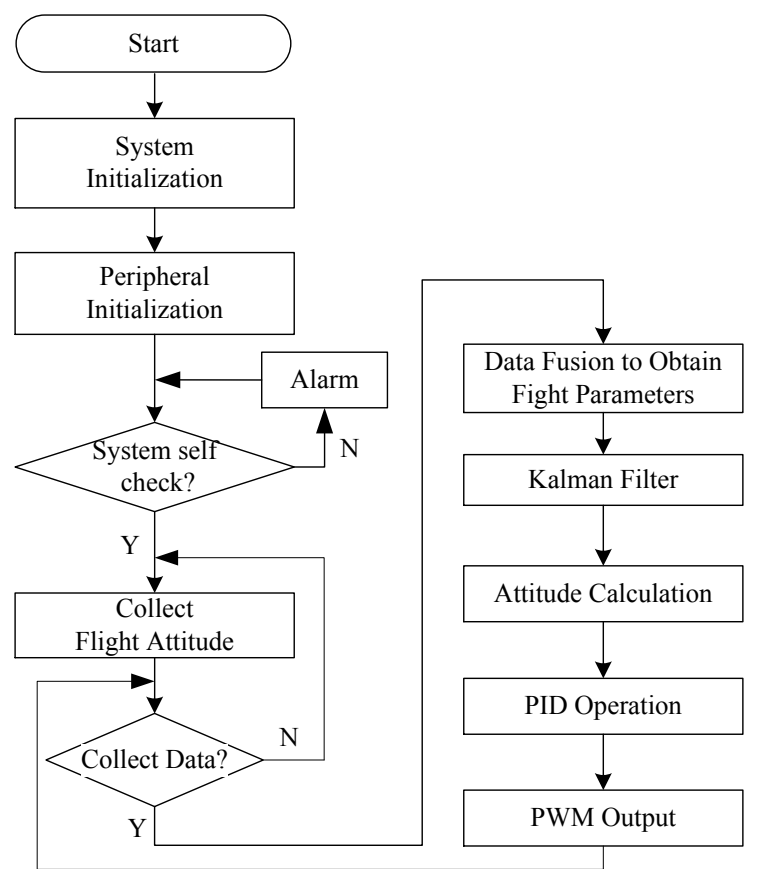

Fig. 6. Diagram of the system software design

\section{Result Analysis and Discussion}

4.1 Parameter adjustment and output curve analysis of PID controller

The control algorithm is the key factor in determining the performance of the quadrotor aircraft. When the quadrotor aircraft is flying and if the external force or electromagnetic interference occurs, the quadrotor aircraft shocks sharply. This system could only depend on control algorithm to recover the stability of the system. Section 3.3 analyzes the PID controller. The reasonable selection of proportion, integral, and differential coefficients is the key for determining the PID controller tracks the flight attitude of the quadrotor aircraft rapidly, adjusts the motor output timely, and recovers the balance of the quadrotor aircraft. The steps of the cascade PID parameter adjustment should follow the rules. The first rule is to adjust the inner loop and then adjust the outer loop. The outer loop is added into the system when the inner loop parameter is determined. For the single loop, the parameter adjustment should follow the principles that the system adjusts the proportion parameter, adjusts the differential parameter, and finally adjusts the integral parameter. The debugging processing is as follows. The first step is to adjust the loop parameters. The integral and differential coefficients are set to zero. The proportion coefficient increases gradually until the output of controlling loop appears in a critical oscillation state. The quadrotor aircraft also appears in a swing phenomenon. The proportional coefficient value is retained. The differential coefficient is set to offset the overshoot reaction of the proportional adjustment in that the quadrotor aircraft tends to be stable. Therefore, adjusting the integral parameters eliminates the static error of the system, and following the 
same steps adjusts the outer loop parameters. The data of the system is tested on a mini quadrotor designed by ourselves. The scene static parameter adjustment testing pattern is shown in Fig. 7. The PID parameters of each adjustment download to the MCU, and quadrotor aircraft flight is started The scene dynamic testing diagrams are shown in Fig. 8(a) and Fig. 8(b). The data of the flight process is transmitted to the host computer through the wireless transmission module. The host computer unin-terruptedly adjusts the parameters of the PID controller according to the flight data to make the flight attitude achieve the best flight state. In this design, the sampling period (100 microseconds) is set, and the roll and pitch angles of the initial state are $0^{\circ}$. Moreover, the yaw angle in the initial state is the angle collected by the microcontroller when the quadrotor works in a state of power-on initia-lization. The pitch angle is $40^{\circ}$ (Each takeoff of quadrotor aircraft takes place in different directions). Based on the above method, the system adjusts repeatedly to obtain the optimum inner and outer loop parameters of the mini quadrotor aircraft, which are shown in Table 1.

The PID controller is set according to the parameters of Table 1 when the quadrotor aircraft is interfered by the stepfunction signal. The control curves of the attitude angles are shown in Fig. 9. Fig. 9(a) demonstrates the step response curve of the pitch angle. The test results show that the maximum error of the pitch angle is $4^{\circ}$, the steady error is within $0.5^{\circ}$, and dynamic response time is approximately 1.3 s. After $1.3 \mathrm{~s}$, the increment of the pitch angle is close to $0^{\circ}$. The system recovers its original equilibrium state. The response curve of the roll angle is shown in Fig. 9(b), wherein the maximum error of roll angle is $6^{\circ}$, and dynamic response time is $2 \mathrm{~s}$. The system tends to be stable after $2 \mathrm{~s}$. Thus, the ranges of the pitch and roll angles show little changes, which indicate that the flight attitude of the quadrotor aircraft is stable. The dynamic adjustment time of yaw angle is slightly longer compared with the pitch and roll angles in Fig. 9(c). The system reaches a stable state in approximately 6 seconds because the adjustment time of the yaw angle has relationships with the value of the system input signal, and the steady-state error of yaw angle is approximately $1^{\circ}$. Based on the above analysis, the system can respond promptly after being disturbed with a short adjustment period and a small overshoot. The system with no shock phenomenon can realize the accuracy of flight attitude conversation and flight stability.

Table 1. Optimum parameters of controllers in pitch, roll, and yaw angle

\begin{tabular}{c|c|c|c|c|c|c}
\cline { 2 - 6 } & $K_{P 1}$ & $K_{I 1}$ & $K_{D 1}$ & $K_{P 2}$ & $K_{I 2}$ & $K_{D 2}$ \\
\hline Pitch Angle & 10.6 & 3.7 & 3.2 & 9.7 & 4.1 & 2.7 \\
Roll Angle & 10.6 & 3.7 & 3.2 & 9.7 & 4.1 & 2.7 \\
Yaw Angle & 8.4 & 4.2 & 3.8 & 8.7 & 3.8 & 3.0 \\
\hline
\end{tabular}

\subsection{Processing and analysis of the flight attitude data of the quadrotor aircraft}

When the mini quadrotor aircraft collects data, angular velocity can be measured directly from the gyroscope sensor, and the angle is integrated by angular velocity. The accuracy of flight attitude is affected by the phenomenon that the mechanical properties of the sensor itself have temperature excursion and zero drift. It can also be influenced by an accumulative error caused by any noise interference in the integration process. This study uses Kalman filter to process the data and correct the data error. Fig. 10 describes the data before and after the acceleration filter. The curve of acceleration data without Kalman filter is shown in Fig. 10(a), and the error of the acceleration data is between -4 and $3 \mathrm{~m} / \mathrm{s}^{2}$. The acceleration data fluctuates greatly, which states that errors by external interference are included in acceleration data. The curve of acceleration data collected by Kalman filter, is shown in Fig. 10(b), and the error of acceleration data is between -0.5 and $0.8 \mathrm{~m} / \mathrm{s}^{2}$. The acceleration data curve becomes smooth and convergent. The fluctuation of acceleration data curve is obviously weakened. Thus, Kalman filter can effectively reduce the data noise and has obvious inhibitory effects on the interference signal.

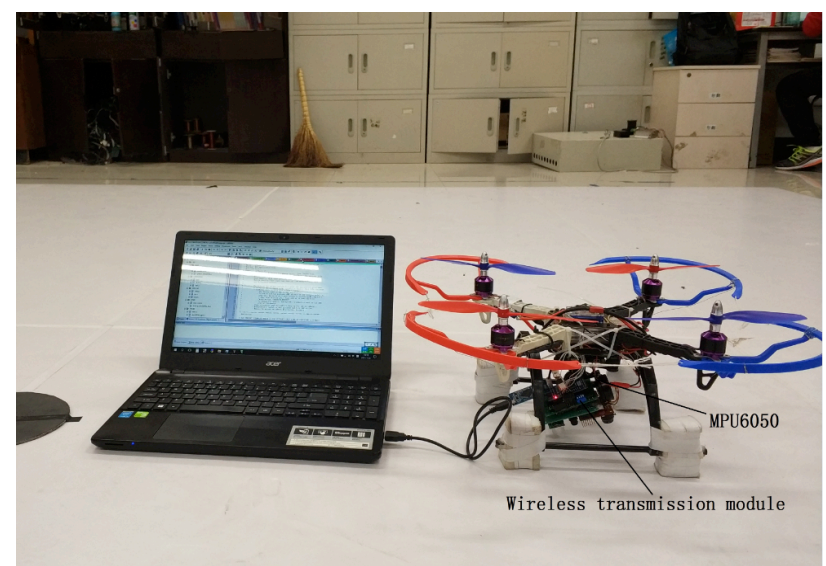

Fig. 7. Scene static parameter adjustment testing diagram

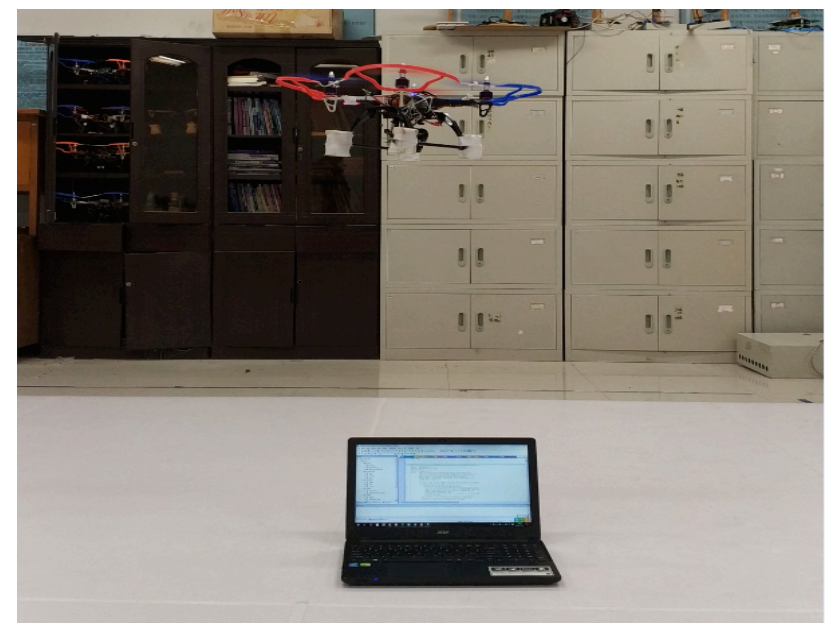

Fig. 8(a). Scene dynamic testing Diagram 1

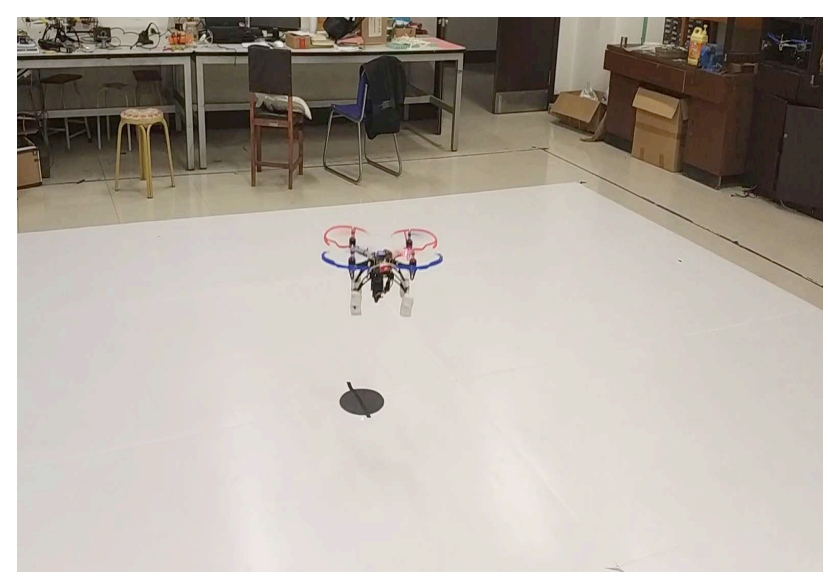

Fig. 8(b). Scene dynamic testing Diagram 2 


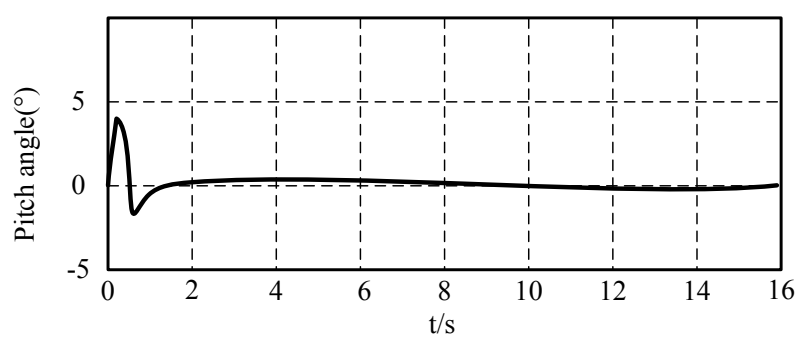

(a)The variation curve of pitch angle

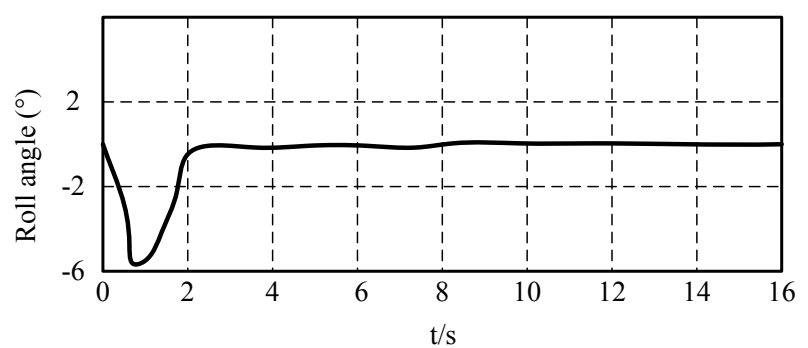

(b) The variation curve of roll angle

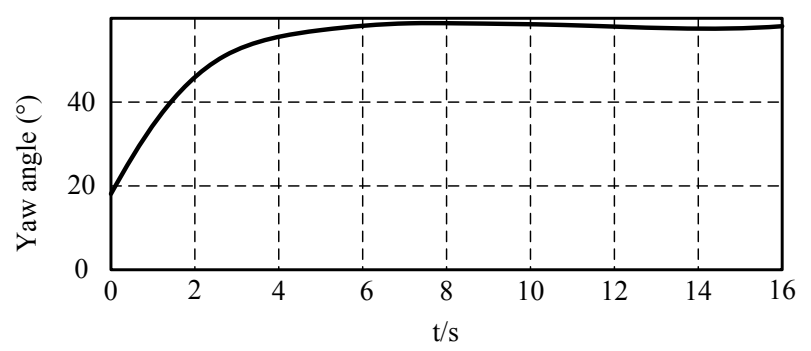

(c) The variation curve of yaw angle

Fig. 9. PID control curve of pitch, roll, and yaw angles

The zero and temperature drifts of the sensors can be analyzed in Tables 2, 3, and 4. The data are shown in Tables 2,3 , and 4 when the quadrotor aircraft is conducting tilt and vertical descent testing. When the quadrotor aircraft is tilted vertically and descended, the theoretical value of acceleration should be $0^{\circ}$, the theoretical value of angular velocity increment is $0^{\circ}$, and the inclination angle in this study is $47^{\circ}$. Tables 2-4 show that the period of measurement is between 289.448 and 389.919 millisecond, the zero error of angular velocity is between $0.061 \%$ s and $0.13 \%$, the zero error of acceleration remains approximately $1.04 \mathrm{~m} / \mathrm{s}^{2}$, and the error of the inclination angle is around $0.036^{\circ}$, which can describe that zero and temperature drifts can be restrained by Kalman filter.

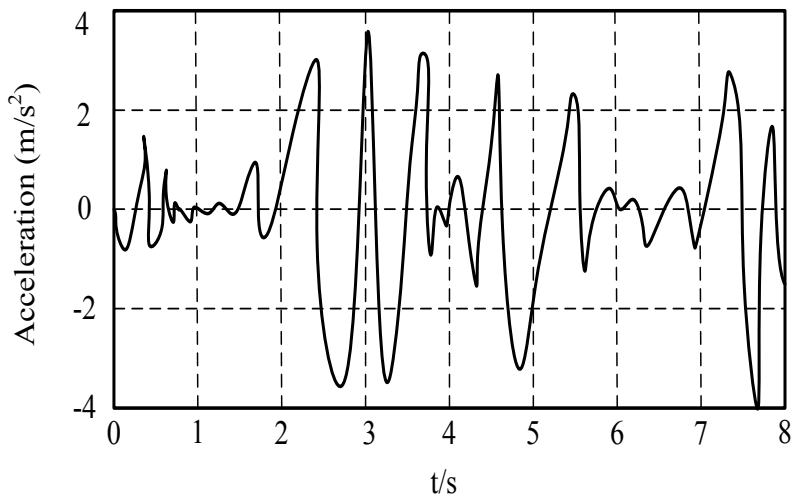

(a) Data before Kalman filter

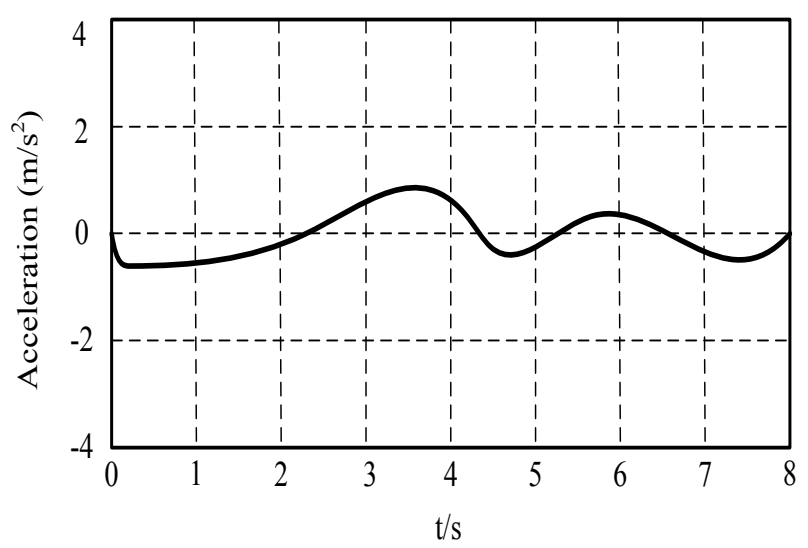

(b) Data after Kalman filter

Fig. 10. Data before and after the acceleration filter

\subsection{Debugging result analysis of DC brushless ESC}

This study uses PWM technology to control the ESC, which can drive the brushless motors and provide the upward force for the four rotors. The speed of the motor is controlled by duty ratio output by ESC and then controls the angular velocity of the four rotors. K60 microprocessor hardware supports the production of PWM and directly regulates the duty ratio. In programming, the duty ratio is controlled by changing the register value in $\mathrm{T} 2$ timer. The duty ratio is between 1200 and 2000. Table 5 shows the data of motor output power testing when the quadrotor aircraft experiences vertical descent and tilts at $47^{\circ}$. Table 5 shows that the motor output force meets the basic linear relationship between $6.5159 x-28.0849$ when the duty ratio of input PWM increases linearly, which indicates a stable flight vehicle.

Table 2. Acceleration testing data

\begin{tabular}{l|l|l|l|l|l|l|l|l|l|l}
\hline Time $/ S$ & $\mathbf{3 8 9 . 4 6 8}$ & $\mathbf{3 8 9 . 5 1 4}$ & $\mathbf{3 8 9 . 5 5 7}$ & $\mathbf{3 8 9 . 6 1 3}$ & $\mathbf{3 8 9 . 6 4 8}$ & $\mathbf{3 8 9 . 7 1 7}$ & $\mathbf{3 8 9 . 7 4 9}$ & $\mathbf{3 8 9 . 8 1 4}$ & $\mathbf{3 8 9 . 8 5 0}$ & $\mathbf{3 8 9 . 9 1 9}$ \\
\hline $\begin{array}{l}\text { Temperature/ } C^{\circ} \\
\mathbf{X} \text { axis- } \\
\text { acceleration } \\
/ \mathrm{m} / \mathrm{s}^{2}\end{array}$ & 31.88 & 31.87 & 31.88 & 31.89 & 31.88 & 31.88 & 31.87 & 31.86 & 31.86 & 31.86 \\
$\begin{array}{l}\mathbf{Y} \\
\text { acceleration } \\
/ \mathrm{m} / \mathrm{s}^{2}\end{array}$ & 0.0200 & 0.0195 & 0.0215 & 0.0190 & 0.0210 & 0.0215 & 0.0176 & 0.020 & 0.0215 & 0.0210 \\
$\begin{array}{l}\mathbf{Z} \\
\text { acceleration } \\
/ \mathrm{m} / \mathrm{s}^{2} \\
\begin{array}{l}\text { Acceleration } \\
/ \mathrm{m} / \mathrm{s}^{2}\end{array}\end{array}$ & 1.0410 \\
$\begin{array}{l}\text { Absolute error } \\
/ \mathrm{m} / \mathrm{s}^{2}\end{array}$ & 1.0414 & 1.0396 & 1.0405 & 1.0391 & 1.0420 & 1.0410 & 1.0391 & 1.0410 & 1.0415 & 1.0415 \\
\hline
\end{tabular}


Table 3. Angle testing data

\begin{tabular}{|c|c|c|c|c|c|c|c|c|c|c|}
\hline Time/ $S$ & 389.468 & 389.514 & 389.559 & 389.613 & 389.659 & 389.719 & 389.761 & 389.815 & 389.843 & 389.895 \\
\hline Temperature / $C^{\circ}$ & 31.88 & 31.87 & 31.88 & 31.89 & 31.87 & 31.87 & 31.87 & 31.86 & 31.86 & 31.86 \\
\hline $\mathrm{X}$ axis angle $/^{\circ}$ & 1.2305 & 1.2360 & 1.2415 & 1.2415 & 1.2469 & 1.2524 & 1.2524 & 1.2579 & 1.2579 & 1.2634 \\
\hline $\mathrm{Y}$ axis angle $/{ }^{\circ}$ & -1.1810 & -1.1755 & -1.1700 & -1.1700 & -1.1700 & -1.1646 & -1.1646 & -1.1591 & -1.1591 & -1.1591 \\
\hline $\mathrm{Z}$ axis angle/ ${ }^{\circ}$ & -47.005 & -47.005 & -47.005 & -47.005 & -47.005 & -47.005 & -47.005 & -47.005 & -47.005 & -47.005 \\
\hline Angle $/^{\circ}$ & -47.035 & -47.035 & -47.035 & -47.035 & -47.036 & -47.003 & -47.003 & -47.036 & -47.036 & -47.036 \\
\hline Absolute error $/^{\circ}$ & 0.0358 & 0.0359 & 0.0359 & 0.0359 & 0.0361 & 0.0026 & 0.0026 & 0.0361 & 0.0361 & 0.0362 \\
\hline
\end{tabular}

Table 4. Angular velocity testing data

\begin{tabular}{|c|c|c|c|c|c|c|c|c|c|c|}
\hline Time/ $s$ & 389.46 & 389.51 & 389.55 & 389.61 & 389.65 & 389.71 & 389.76 & 389.81 & 389.84 & 389.890 \\
\hline Temperature $/ C^{\circ}$ & 31.88 & 31.87 & 31.88 & 31.89 & 31.87 & 31.87 & 31.87 & 31.86 & 31.86 & 31.86 \\
\hline $\begin{array}{l}\mathbf{X} \text { axial angular velocity } \\
/ \circ / \mathrm{s}\end{array}$ & -0.061 & 0.000 & 0.000 & -0.122 & -0.061 & -0.061 & -0.061 & -0.061 & 0.000 & -0.061 \\
\hline $\begin{array}{l}\mathrm{Y} \text { axial angular velocity } \\
/ \mathrm{O} / \mathrm{s}\end{array}$ & 0.000 & 0.061 & 0.061 & 0.061 & 0.061 & 0.061 & 0.000 & 0.000 & 0.000 & 0.000 \\
\hline $\begin{array}{l}\mathrm{Z} \text { axial angular velocity } \\
/ \% / \mathrm{s}\end{array}$ & 0.061 & 0.000 & 0.000 & 0.000 & 0.000 & 0.000 & 0.000 & 0.000 & 0.061 & 0.000 \\
\hline Angular velocity $/ \% / \mathrm{s}$ & 0.0863 & 0.0610 & 0.0610 & 0.136 & 0.0863 & 0.0863 & 0.061 & 0.061 & 0.061 & 0.061 \\
\hline Absolute error $/{ }^{\circ} / \mathrm{s}$ & 0.0102 & 0.0151 & 0.0151 & 0.0599 & 0.0102 & 0.0102 & 0.0151 & 0.0151 & 0.0151 & 0.0151 \\
\hline
\end{tabular}

Table 5. Motor output force testing data

\begin{tabular}{l|l|l|l|l|l|l|l|l|l|l}
\hline Battery voltage/V & $\mathbf{1 1 . 5 1 4}$ & $\mathbf{1 1 . 4 8 6}$ & $\mathbf{1 1 . 4 5 9}$ & $\mathbf{1 1 . 4 3 1}$ & $\mathbf{1 1 . 4 0 4}$ & $\mathbf{1 1 . 3 7 6}$ & $\mathbf{1 1 . 3 4 8}$ & $\mathbf{1 1 . 3 2 0}$ & $\mathbf{1 1 . 2 6 3}$ & $\mathbf{1 1 . 2 6 5}$ \\
\hline PWM=1200+(n-1)/ \% & 1 & 10 & 20 & 30 & 40 & 50 & 60 & 70 & 80 & 90 \\
Lifting force /g & 16 & 54 & 103 & 158 & 210 & 275 & 350 & 430 & 510 & 584 \\
\hline
\end{tabular}

\section{Conclusions}

The aim of this study was to determine the flight attitude stability and system response lag time of a mini quadrotor aircraft. Thus, a new type of quadrotor aircraft based on the K60 microprocessor was proposed. First, software and hardware circuits were designed, and system stability was analyzed for the quadrotor aircraft affected by noise interference. Then, the Euler equation model of the quadrotor aircraft was established, and data integration was adopted. Finally, the experimental study was carried out. The following conclusions can be drawn:

(1) Noise inhibition in the mini quadrotor aircraft system is dependent on gyroscope accuracy. The Kalman filter can restrain noise and eliminate interference. Subsequently, accurate data on flight attitude angle is obtained and reliable autonomous flight is ensured.

(2) The stability of the mini quadrotor aircraft is related to the adjusted algorithm of the system. The cascade dual closed-loop PID control algorithm, which can be employed in three ways, is designed to ensure motor output force stability and reduce the fluctuation range of the quadrotor aircraft.

(3) The output response of the quadrotor aircraft is proportional to the response speed of the brushless motor. The output linearization of the brushless motor accelerates the output response speed of the quadrotor aircraft.

This study, which combined experimental and theoretical work, integrated the design ideas and implementation process of the quadrotor aircraft. The filtering design and the cascade dual closed-loop PID control algorithm were effective and practical, and both can be used as reference for the study and development of mini quadrotor aircraft. However, the air data with low Reynolds number used considered for this study were lacking. In the future, the system design will be combined with the air-assisted model, which hopefully can effectively correct the attitude of the mini quadrotor aircraft.

This is an Open Access article distributed under the terms of the Creative Commons Attribution Licence

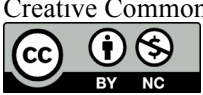

\section{References}

1. Liu, Y. S., Yang, S. G., Wang, W., “Active disturbance rejection flight control method for four-rotor aircraft". Journal of Control theory and Application, 32(10), 2015, pp. 1351-1360.

2. Bouabdallah, S., Noth, A., Siegwart, R., "PID VS LQ control techniques applied to an indoor micro-quadrotor". In: IEEE International Conference on Intelligent Robots and Systems (IROS), Sendai, Japan: IEEE, 2004, pp. 2451-2456.
3. Nie, B., Ma, H. X., "Research status and key technologies of micro aircraft". Journal of Electronics Optics and Control, 14(6), 2007, pp. 113-117.

4. Ghosh, A., Incel, D., Kumar, V. S., "Multichannel scheduling and spanning trees: Throughput-delay trade-off for fast data collection in sensor networks". IEEE ACM Transactions on Networking, 19(6), 2011, pp. 1731-1744. 
5. Gelal, E., Jakllari, G., Krishnamurthy, S. V., "Topology control to simultaneously achieve near-optimal node degree and low path stretch in Ad Hoc networks". In: 2006 3rd Annual IEEE Communications Society on Sensor and Ad Hoc Communications and Networks(SECON), Reston, USA: IEEE, 2006, pp. 43-439.

6. Zhou, M., Xu, G, H., Luo, Z. K., "Adaptive single neuron PID control of four rotor aircraft". Journal of Military communication technology, 9(3), 2013, pp. 7-11.

7. Wei, L, W., "Design of four rotor aircraft control system". Master thesis of Harbin Institute of Technology, China, 2010, pp. 1-72.

8. Wang, L. X., "Design of Attitude Control Based on Sliding Mode Control Theory with Quadrotor". Master thesis of Northeastern University, 2009, pp. 1-70.

9. Xu, J. K., Gong, H. S., “A underactuated VTOL aircraft sliding mode control input saturation". Journal of electrical machinery and control, 3(17), 2013, pp. 92-97.

10. $\mathrm{Wu}, \mathrm{C} ., \mathrm{Xu}, \mathrm{J} . \mathrm{B}$., “Anti disturbance control of trajectory tracking for four-rotor aircraft". Journal of Control theory and Applications, 3(11), 2016, pp. 1422-1430.

11. Mckerrow, P., "Modelling the draganflyer four-rotor helicopter". In: Proceedings of IEEE International Conference on Robotics and Automation, New Orleans, USA: IEEE, 2004, pp. 3596-3601.

12. Li, X. N., "Adaptive PID four-rotor aircraft control based on RBF neural network". Journal of Control Engineering, 2016, pp. 378382 .

13. Yan, H. W., Kun, P. H., "Trajectory Tracking Control of a Quadrotor UAV". Journal of Applied Mechanics and Materials, 2013, 2715(419), pp. 718-724.

14. Rao, K. Q., "Attitude estimation method for four rotor aircraft". Journal of Photoelectricity and Control, 22(5), 2015, pp. 25-28.

15. Roberta, V. G., "Unscented Kalman filter for spacecraft attitude estimation using modified Rodrigues parameters and real data". Journal of Computational and Applied Mathematics, 35(3), 2016, pp. $835-846$.
16. $\mathrm{Xu}, \mathrm{Z}$., "Research on wind resistance of four rotor UAV Based on SMC". Journal of Photoelectricity and Control, 1(24), 2017, pp. 67-71.

17. Liu, J. Y., Jia, W. F., Lai, J. Z., "Multi information nonlinear fusion navigation method for mini four-rotor aircraft and its implementation". Journal of Nanjing University of Aeronautics and Astronautics, 45(5), 2013, pp. 575-582.

18. Mahony, R., Kumar, V., Corke, P., "Multirotor aerial vehicles: modeling". Journal of Estimation and Control of Quadrotor,19(3), 2012, pp. 20-23.

19. DAS, A., Lewis, F., Subbarao, K., "Backstepping approach for controlling a quadrotor using lagrange form dynamics". Journal of Intelligent and Robotic Systems, 56(1/2), 2009, pp. 127-151.

20. Lv, Q., Ni, P. P., Wang, G. S., Liu, F., "Hovering correction of quadrotor aircraft based on optical flow sensor". Journal of the Academy of Armored Forces Engineering, 28(3), 2014, pp. 68-72.

21. He, R. J., Bachrach, A., Achtelik, M., "On the design and use of a micro air vehicle to track and avoid adversaries". International Journal of Robotics Research, 29(5), 2010, pp. 529-546.

22. Lin, X. M., Wang, C., "Design of adaptive robust sliding mode controller for four rotor aircraft". Journal of Instrumentation, 36(7), 2015, pp.1522-1528.

23. Sun, J. L., Liu, C. S., Lu, K., “Optimal Robust Control for Attitude of Quadrotor Aircraft Based on SDRE". In: 2015 34th Chinese Control Conference, Hangzhou, China: IEEE, 2015, pp. 2333-2337.

24. Schreier, M., Darmstadt, T., "Modeling and adaptive control of a quadrotor". In: 2012 IEEE International Conference on Mechatronics and Automation, Chengdu, China: IEEE, 2012, pp. 383-390.

25. Wang, W., Qiu, Q., “Aircraft modeling and position tracking control of six rotors". Journal of Electronic Devices, 37(3), 2014, pp. 507-512.

26. Wang, X. X., Liang, Y., Pan, Q., "Nonlinear system for colored measurement noise Unscented Kalman filter". Journal of Automation, 38(6), 2012, pp. 986-988. 